\title{
THE WEIGHTED SOBOLEV AND MEAN VALUE INEQUALITIES
}

\author{
ADRIANO ALVES DE MEDEIROS \\ (Communicated by Lei Ni) \\ Dedicated to my son João Gabriel
}

\begin{abstract}
In this paper we prove a Michael-Simon inequality in the weighted setting and using this inequality we obtain a diameter control depending of the $f$-mean curvature, which is based in the work of Topping.
\end{abstract}

\section{INTRODUCTION}

The classical Sobolev inequality, proved by S. L. Sobolev in the celebrated paper [13], states that for any smooth function on $\mathbb{R}^{n}$ with compact support $u \in C_{0}^{\infty}\left(\mathbb{R}^{n}\right)$ and $1<p<n$ there exists a constant $S=S(p, n)>0$ such that

$$
\left(\int_{\mathbb{R}^{n}}|u|^{\frac{n p}{n-p}} d x\right)^{\frac{n-p}{n p}} \leq S(p, n) \cdot\left(\int_{\mathbb{R}^{n}}|D u|^{p} d x\right)^{\frac{1}{p}} .
$$

The Sobolev inequality (1.1) also holds for $p=1$ by the Gagliardo-Nirenberg estimates, see [18. Its numerous versions play a central role in mathematics, specially in the theory of partial differential equations, mathematical analysis, mathematical physics, and differential geometry as one can glimpse in the three excellent volumes, edited by Vladimir Maz'ya, 8]. The Sobolev inequality was extended to minimal hypersurfaces of $\mathbb{R}^{n}$ by M. Miranda [10] and refined in 2] to derive a priori gradient bounds of solutions to the minimal surface equation. The next step was taken by J. Michael and L. Simon in [9], where they proved a version of the Sobolev inequality valid for a general class of measures and subsets of $\mathbb{R}^{n}$ and of particular interest in the study of surfaces of prescribed mean curvature. The Sobolev inequality has been generalized to general Riemannian submanifolds by D. Hoffman and J. Spruck [5] in the following theorem.

Theorem 1.1 (Hoffman-Spruck, [5]). Let $\varphi: M \rightarrow \bar{M}$ be an isometric immersion of a Riemannian m-manifold $M$ into the Riemannian $n$-manifold $\bar{M}$ with mean curvature vector $H$ and injectivity radius $\bar{R}=\inf _{p \in M} \operatorname{dist}_{\bar{M}}(p, \operatorname{cut}(\bar{M}))$. Suppose that the sectional curvature $K_{\bar{M}} \leq b, b \in \mathbb{R} \backslash\{0\}$. Let $h \in C^{1}(M)$ be a nonnegative function vanishing in $\partial M$. Let

$$
\rho_{0}= \begin{cases}(\sqrt{b})^{-1} \sin ^{-1}(\sqrt{b})(1-\alpha)^{-1 / m}\left(\omega_{m}^{-1} \operatorname{vol}(\operatorname{supp} h)\right)^{1 / m} & \text { if } b>0 \\ (1-\alpha)^{-1 / m}\left(\omega_{m}^{-1} \operatorname{vol}(\operatorname{supp} h)\right)^{1 / m} & \text { if } \quad b<0\end{cases}
$$

Received by the editors April 17, 2013 and, in revised form, May 24, 2013.

2010 Mathematics Subject Classification. Primary 53C42; Secondary 53C21.

The author would like to thank Gregorio Pacelli Bessa and Jorge Herbert Soares de Lira for stimulating conversations about this subject.

(C)2014 American Mathematical Society 
If $2 \rho_{0} \leq \bar{R}$ and $b(1-\alpha)^{-2 / m}\left(\omega_{m}^{-1} \operatorname{vol}(\operatorname{supp} h)\right)^{2 / m} \leq 1$, then

$$
\left(\int_{M} h^{m /(m-1)} d v\right)^{(m-1) / m} \leq c(m) \int_{M}(|\nabla h|+h|H|) d v
$$

where $c(m)$ is a constant depending on $m, \omega_{m}$ is the volume of the unit ball in $\mathbb{R}^{m}$.

In this note we are concerned with establishing a weighted version of the MichaelSimon inequality. An $m$-dimensional weighted manifold is a triple $M_{f}=\left(M, g, d v_{f}\right)$, where $(M, g)$ is a Riemannian manifold, $f: M \rightarrow \mathbb{R}$ is a selected smooth function on $M$ and $d v_{f}=e^{-f} d v$ is the weighted measure, where $d v$ denotes the Riemannian measure of $(M, g)$. There has been an increasing interest in the geometric analysis on weighted manifolds and it is important to search for these results since one has the flexibility to change the measure without changing the underlying manifold. In this spirit our version of the Michael-Simon Sobolev inequality is stated in the following theorem.

Theorem 1.2 (Weighted Michael-Simon Inequality). Let $\mathbb{R}_{f}^{n}=\left(\mathbb{R}^{n}, g, d_{f} v\right)$ be a weighted Riemannian manifold modeled upon $\mathbb{R}^{n}$ with the Euclidean metric structure $g$. So, there exists $c_{k}$ depending only on $k$ so that if $u>0$ is a Lipschitz function with compact support on a submanifold $\Sigma^{k} \subset \mathbb{R}^{n}$ and

$$
\int_{\Sigma}\langle x, \bar{\nabla} f\rangle d_{f} v \leq 0 \text { on } \Sigma
$$

then

$$
\left(\int_{\Sigma} u^{\frac{k}{k-1}} d_{f} v\right)^{\frac{k-1}{k}} \leq c_{k} \int_{\Sigma}\left[\left|\nabla^{\Sigma} u\right|+u\left|\mathbf{H}_{f}\right|\right] d_{f} v .
$$

The proof of our result is based on the $f$-mean value inequality and a covering lemma also used by Michael and Simon.

As we mentioned earlier, there are several applications of the Sobolev inequality. A direct consequence is the following weighted isoperimetric inequality.

Theorem 1.3. Let $\Omega \subset \Sigma$ be open with Lipschitz boundary $\partial \Omega$. If (1.2) holds, then we have the inequality

$$
V_{f}(\Omega)^{\frac{k-1}{k}} \leq c_{k}\left[V_{f}(\partial \Omega)+\int_{\Omega}\left|\mathbf{H}_{f}\right|\right] .
$$

Another application that we would like to highlight can be found in Topping [16], where the author obtains an intrinsic diameter control depending of the mean curvature. Here, we use the weighted Sobolev inequality for generalizing this result in the weighted setting.

Theorem 1.4. Let $\Sigma^{k} \hookrightarrow \mathbb{R}_{f}^{n}$ be a closed connected submanifold. If (1.2) occurs, then we have the following estimate for the intrinsic diameter $d_{\text {int }}$ :

$$
d_{i n t} \leq C(n, f) \int_{\Sigma}\left|\mathbf{H}_{f}\right|^{k-1} d_{f} v .
$$

Remark. We would like to mention some related works by M. Rimoldi and D. Impera [6] and M. Batista and H. Mirandola [11] where similar inequalities had been proved using another techniques and substantially distinct applications from ours were presented. 


\section{Preliminaries}

The $f$-Laplacian associated with the weighted manifold $M_{f}$ is the operator

$$
\Delta_{f} u=\operatorname{div}_{f}(\nabla u):=e^{f} \operatorname{div}\left(e^{-f} \nabla u\right)=\Delta u-\langle\nabla u, \nabla f\rangle,
$$

which is symmetric on $L^{2}\left(M, d_{f} v\right)$. Hereafter, $B_{r}(p)$ and $\partial B_{r}(p)$ will denote the geodesic ball and sphere of $(M, g)$ of radius $r>0$ centered at $p \in M$, and we also set

$$
\operatorname{vol}_{f}\left(B_{r}(p)\right)=\int_{B_{r}(p)} e^{-f} d v, \quad \operatorname{vol}_{f}\left(\partial B_{r}(p)\right)=\int_{\partial B_{r}(p)} e^{-f} d S,
$$

where $d S$ stands for the $(m-1)$-Hausdorff measure.

Proposition 2.1. Let $\phi: \Sigma^{n} \rightarrow N^{n+1}$ be a hypersurface and let $\Phi:(-\epsilon, \epsilon) \times \Sigma \rightarrow N$ be a proper variation of $\phi$ with normal variational vector field $V$. If $\mathbf{H}$ is the mean curvature vector, then

$$
\left.\frac{d}{d t}\right|_{t=0} \operatorname{vol}_{f}(\Phi(t, \Sigma))=-\int_{\Sigma}\langle\bar{\nabla} f+n H N, V\rangle e^{-f} d v .
$$

Proof. It is well known that

$$
\left.\frac{d}{d t}\right|_{t=0} \int_{\Sigma} d v_{t}=-\int_{\Sigma} n\langle H, V\rangle d v
$$

Furthermore,

$$
\frac{d}{d t}\left[e^{-f\left(\Phi_{t}\right)}\right]=-e^{-f}\langle\bar{\nabla} f, V\rangle
$$

Therefore,

$$
\left.\frac{d}{d t}\right|_{t=0} \operatorname{vol}_{f}(\Phi(t, \Sigma))=-\int_{\Sigma}\langle\bar{\nabla} f+n H, V\rangle e^{-f} d v .
$$

This finishes the proof of the proposition.

Motivated by the previous proposition, we define the $f$-mean curvature vector field by

$$
\mathbf{H}_{f}=\mathbf{H}+(\bar{\nabla} f)^{\perp},
$$

where $\perp$ denotes the projection on the normal bundle. Consequently, we say that an immersed submanifold is $f$-minimal if $\mathbf{H}_{f}=0$.

\section{The mean value inequality}

In this section, we prove the mean value inequality in the weighted setting.

Proposition 3.1 (The mean value inequality). Let $\left(\mathbb{R}^{n}, g, d_{f} v\right)$ a weighted Riemannian manifold. If $\Sigma^{k} \subset \mathbb{R}^{n}$ is an $f$-minimal submanifold and $u$ is a function on $\Sigma$, then

$$
t^{-k} \int_{B_{t} \cap \Sigma} u d_{f} v-s^{-k} \int_{B_{s} \cap \Sigma} u d_{f} v=\int_{\left(B_{t} \backslash B_{s}\right) \cap \Sigma} u \frac{\left|x^{\perp}\right|^{2}}{\left|x^{T}\right|^{k+2}} d_{f} v-A,
$$

where

$$
A=\int_{s}^{t} \tau^{-k-1}\left[\int_{B_{\tau} \cap \Sigma}\left\{u\langle\bar{\nabla} f, x\rangle-\left(\tau^{2}-|x|^{2}\right) \Delta_{f}^{\Sigma} u\right\} d_{f} v\right] d \tau
$$


Proof. We have

Therefore,

$$
\left\langle\nabla^{\Sigma}|x|^{2}, w\right\rangle=\left\langle\bar{\nabla}|x|^{2}, w\right\rangle=2\langle x, w\rangle
$$

$$
\nabla^{\Sigma}|x|^{2}=2 x^{T}
$$

We conclude that

$$
\begin{aligned}
\Delta_{\Sigma}|x|^{2}=\left\langle\nabla_{e_{i}}^{\Sigma} \nabla^{\Sigma}|x|^{2}, e_{i}\right\rangle & =2\left\langle\nabla_{e_{i}}^{\Sigma} x^{T}, e_{i}\right\rangle \\
& =2\left[\left\langle\nabla_{e_{i}}^{\Sigma} x, e_{i}\right\rangle-\left\langle\nabla_{e_{i}}^{\Sigma} x^{\perp}, e_{i}\right\rangle\right] \\
& =2 k+2\left|x^{\perp}\right| H \\
& =2 k+2\left|x^{\perp}\right|\left(H_{f}-(\bar{\nabla} f)^{\perp}\right) .
\end{aligned}
$$

Since $\Sigma$ is an $f$-minimal submanifold, we have

$$
\Delta_{\Sigma}|x|^{2}=2 k-2\left|x^{\perp}\right|(\bar{\nabla} f)^{\perp} .
$$

In view of this, we have

$$
\Delta_{f}^{\Sigma}|x|^{2}=\Delta_{\Sigma}|x|^{2}-\left\langle\nabla^{\Sigma}|x|^{2}, \nabla^{\Sigma} f\right\rangle=2 k-2\left|x^{\perp}\right|(\bar{\nabla} f)^{\perp}-2\left\langle x^{T}, \nabla^{\Sigma} f\right\rangle .
$$

Using this we obtain

(3.3) $2 k \int_{B_{s} \cap \Sigma} u d_{f} v=\int_{B_{s} \cap \Sigma} u \Delta_{f}^{\Sigma}|x|^{2} d_{f} v+2 \int_{B_{s} \cap \Sigma} u\left(\left|x^{\perp}\right|(\bar{\nabla} f)^{\perp}+\left\langle\nabla^{\Sigma} f, x^{T}\right\rangle\right) d_{f} v$.

Note that, using the Stokes theorem, we get

$$
\begin{aligned}
\int_{B_{s} \cap \Sigma} u \Delta_{f}^{\Sigma}|x|^{2} d_{f} v & =\int_{B_{s} \cap \Sigma} \operatorname{div}_{f}\left(u \nabla^{\Sigma}|x|^{2}\right) d_{f} v-2 \int_{B_{s} \cap \Sigma}\left\langle\nabla^{\Sigma} u, \nabla^{\Sigma}|x|^{2}\right\rangle d_{f} v \\
& =\int_{\partial B_{s} \cap \Sigma}\left\langle u \nabla^{\Sigma}|x|^{2}, \nu\right\rangle d_{f} S+2 \int_{B_{s} \cap \Sigma}|x|^{2} \Delta_{f}^{\Sigma} u d_{f} v-2 s^{2} \int_{\partial B_{s} \cap \Sigma}\left\langle\nabla^{\Sigma} u, \nu\right\rangle \\
& =2 \int_{\partial B_{s} \cap \Sigma} u\left|x^{T}\right| d_{f} S+2 \int_{B_{s} \cap \Sigma}\left(|x|^{2}-s^{2}\right) \Delta_{f}^{\Sigma} u d_{f} v .
\end{aligned}
$$

Furthermore, it follows from the co-area formula that

$$
\int_{B_{s} \cap \Sigma} u e^{-f} d v=\int_{\{r \leq s\}} \frac{u e^{-f}\left|\nabla^{\Sigma} r\right|}{\left|\nabla^{\Sigma} r\right|} d v=\int_{-\infty}^{s} \int_{r=\tau} \frac{u e^{-f}}{\left|\nabla^{\Sigma} r\right|} d \tau=\int_{-\infty}^{s} \int_{r=\tau} \frac{u e^{-f}|x|}{\left|x^{T}\right|} d \tau,
$$

which implies that

$$
\frac{d}{d s}\left(\int_{B_{s} \cap \Sigma} u d_{f} v\right)=s \int_{\partial B_{s}} \frac{u}{\left|x^{T}\right|} d_{f} v .
$$

Now, using (3.3), (3.4) and (3.5), we obtain

$$
\begin{aligned}
\frac{d}{d s}\left(s^{-k} \int_{B_{s} \cap \Sigma} u d_{f} v\right) & =-\frac{k}{s^{k+1}} \int_{B_{s} \cap \Sigma} u d_{f} v+\frac{1}{s^{k}} \frac{d}{d s}\left(\int_{B_{s} \cap \Sigma} u d_{f} v\right) \\
& =-\frac{1}{s^{k+1}}\left[\int_{\partial B_{s} \cap \Sigma} u\left|x^{T}\right| d_{f} S+\int_{B_{s} \cap \Sigma} u\langle\bar{\nabla} f, x\rangle d_{f} v\right] \\
& -\frac{1}{s^{k+1}} \int_{B_{s} \cap \Sigma}\left(|x|^{2}-s^{2}\right) \Delta_{f}^{\Sigma} u d_{f} v+\frac{1}{s^{k}} \int_{\partial B_{s} \cap \Sigma} \frac{|x|}{\left|x^{T}\right|} u d_{f} S \\
& =\frac{1}{s^{k+1}} \int_{\partial B_{s} \cap \Sigma} \frac{\left|x^{\perp}\right|^{2}}{\left|x^{T}\right|^{2}} u d_{f} S-\frac{1}{s^{k+1}} \int_{B_{s} \cap \Sigma} u\langle\bar{\nabla} f, x\rangle d_{f} v \\
& -\int_{B_{s} \cap \Sigma}\left(|x|^{2}-s^{2}\right) \Delta_{f}^{\Sigma} u d_{f} v .
\end{aligned}
$$

Integrating and using the co-area formula prove the statement. 
The next lemma is an important tool that we will use in the proof of the weighted Michael-Simon Inequality.

Lemma 3.2. Let $\Sigma^{k} \subset \mathbb{R}_{f}^{n}$ be a submanifold with the $f$-mean curvature $H_{f}$ and let $u$ be a nonnegative function on $\Sigma$. Then for $s<t$,

$t^{-k} \int_{B_{t} \cap \Sigma} u d_{f} v-s^{-k} \int_{B_{s} \cap \Sigma} d_{f} v \geq \int_{s}^{t} \tau^{-k-1}\left[\int_{B_{\tau} \cap \Sigma}\left\langle x, \nabla^{\Sigma} u+u\left(\mathbf{H}_{\mathbf{f}}-\bar{\nabla} f\right)\right\rangle d_{f} v\right] d \tau$.

Proof. As in the previous proof, we have

$$
2 k \int_{B_{s} \cap \Sigma} u d_{f} v=\int_{B_{s} \cap \Sigma} u \Delta_{f}^{\Sigma}|x|^{2} d_{f} v+2 \int_{B_{s} \cap \Sigma} u\left[\left|x^{\perp}\right|\left((\bar{\nabla} f)^{\perp}-H_{f}\right)+\left\langle\nabla^{\Sigma} f, x^{T}\right\rangle\right] d_{f} v .
$$

Therefore,

$$
\begin{aligned}
\frac{d}{d s}\left(s^{-k} \int_{B_{s} \cap \Sigma} u d_{f} v\right) & =-\frac{k}{s^{k+1}} \int_{B_{s} \cap \Sigma} u d_{f} v+\frac{1}{s^{k}} \frac{d}{d s}\left(\int_{B_{s} \cap \Sigma} u d_{f} v\right) \\
& =-\frac{1}{s^{k+1}} \int_{B_{s} \cap \Sigma} u\left[\left|x^{\perp}\right|\left((\bar{\nabla} f)^{\perp}-H_{f}\right)+\left\langle\nabla^{\Sigma} f, x^{T}\right\rangle\right] d_{f} v \\
& +\frac{1}{s^{k}} \int_{\partial B_{s} \cap \Sigma} u \frac{|x|}{\left|x^{T}\right|}-\frac{1}{2 s^{k+1}} \int_{B_{s} \cap \Sigma} u \Delta_{f}^{\Sigma}|x|^{2} d_{f} v
\end{aligned}
$$

Now, using Green's Formula, we get

$$
\int_{B_{s} \cap \Sigma} u \Delta_{f}^{\Sigma}|x|^{2} d_{f} v=\int_{\partial B_{s} \cap \Sigma} u\left\langle\nabla^{\Sigma}|x|^{2}, \nu\right\rangle d_{f} S-\int_{B_{s} \cap \Sigma}\left\langle\nabla^{\Sigma} u, \nabla^{\Sigma}|x|^{2}\right\rangle d_{f} v .
$$

By the previous calculus, we have

$$
\frac{1}{s^{k}} \int_{\partial B_{s} \cap \Sigma} u \frac{|x|}{\left|x^{T}\right|}-\frac{1}{2 s^{k+1}} \int_{\partial B_{s} \cap \Sigma} u\left\langle\nabla^{\Sigma}|x|^{2}, \nu\right\rangle d_{f} S \geq 0 .
$$

Applying this in the inequality above, we obtain

$$
\frac{d}{d s}\left(s^{-k} \int_{B_{s} \cap \Sigma} u d_{f} v\right) \geq \frac{1}{s^{k+1}} \int_{B_{s} \cap \Sigma}\left\langle x, \nabla^{\Sigma} u+u\left(\mathbf{H}_{\mathbf{f}}-\bar{\nabla} f\right)\right\rangle d_{f} v .
$$

Integrating, we prove the statement.

Corollary 3.3. On the assumptions of the previous lemma, if

$$
\int_{\Sigma}\langle x, \bar{\nabla} f\rangle \leq 0 \text { on } \Sigma
$$

then

$$
t^{-k} \int_{B_{t} \cap \Sigma} u d_{f} v-s^{-k} \int_{B_{s} \cap \Sigma} d_{f} v \geq \int_{s}^{t} \tau^{-k-1}\left[\int_{B_{\tau} \cap \Sigma}\left\langle x, \nabla^{\Sigma} u+u \mathbf{H}_{\mathbf{f}}\right\rangle d_{f} v\right] d \tau
$$

\section{Weighted Michael-Simon Inequality}

In order to prove the weighted Michael-Simon Inequality we need three lemmas. The proof of these results may be found in [3]. The first result is a covering lemma. 
Lemma 4.1. If $\mathcal{B}$ is a family of closed balls in a metric space with

$$
\sup \{\operatorname{diam}(B) \mid B \in \mathcal{B}\}<\infty,
$$

then there is a pairwise disjoint subcollection $\mathcal{B}^{\prime} \subset \mathcal{B}$ so that

$$
\bigcup_{B \in \mathcal{B}} \subset \bigcup_{B \in \mathcal{B}^{\prime}} 5 B
$$

where $5 B$ is the ball with the same center as $B$ but with 5 times the radius.

Lemma 4.2. Suppose $f, g \geq 0$ are bounded, nondecreasing, with

$$
1 \leq \limsup _{\sigma \rightarrow 0} \frac{f(\sigma)}{\sigma^{k}}
$$

and for any $0<\sigma<\rho$,

$$
\frac{f(\sigma)}{\sigma^{k}} \leq \frac{f(\rho)}{\rho^{k}}+\int_{0}^{\rho} t^{-k} g(t) d t .
$$

Set $f_{\infty}=\lim _{t \rightarrow \infty} f(t)$ and $\rho_{0}=2\left(f_{\infty}\right)^{1 / k}$. Then there exists $\rho \in\left(0, \rho_{0}\right)$ with

$$
f(5 \rho) \leq \frac{5^{k}}{2} \rho_{0} g(\rho) .
$$

Lemma 4.3. Suppose that $\nu$ is a measure on a space $X, f \geq 0$ is in $L^{1}(\nu)$, $A_{t}=\{x \mid f(x)>t\}$, and $\alpha>0$. Then

$$
\frac{1}{\alpha} \int f^{\alpha} d \nu=\int_{0}^{\infty} t^{\alpha-1} \nu\left(A_{t}\right) d t
$$

It follows that if $t_{0} \geq 0$, then

$$
\frac{1}{\alpha} \int_{A_{t_{0}}}\left(f^{\alpha}-t_{0}^{\alpha}\right) d \nu=\int_{t_{0}}^{\infty} t^{\alpha-1} \nu\left(A_{t}\right) d t .
$$

Now, we are able to prove the weighted Michael-Simon inequality. In fact, we prove the following slightly more general inequality than (1.3).

Theorem 4.4. Let $\Sigma^{k} \subset \mathbb{R}_{f}^{n}$ be a submanifold. If $u>0$ is a Lipschitz function with compact support on $\Sigma$, then there exists a constant $c_{k}$ depending only on $k$ satisfying

$$
\left(\int_{\Sigma} u^{\frac{k}{k-1}} d_{f} v\right)^{\frac{k-1}{k}} \leq c_{k} \int_{\Sigma}\left[\left|\nabla^{\Sigma} u\right|+u\left|\mathbf{H}_{\mathbf{f}}-\bar{\nabla} f\right|\right] d_{f} v .
$$

Remark 4.5. The particular form stated in Theorem 1.3 may be proved by minor modification in the proof of the general case using hypothesis (1.2). In this case, combining (4.6) and Corollary 3.3 yield (1.3).

Proof. Let $\Sigma \subset \mathbb{R}^{n}$ be a submanifold and define $J(r)$ by

$$
J(r)=\frac{1}{\omega_{k} r^{k}} \int_{B_{r} \cap \Sigma} h d_{f} v
$$

where $\omega_{k}=\int_{B_{1}} d_{f} v$ and $h$ is a nonnegative function with compact support. Therefore, using the mean value inequality in Lemma 3.2 and the Cauchy-Schwarz inequality, we obtain

$$
r^{k+1} J^{\prime}(r) \geq \frac{1}{\omega_{k}} \int_{B_{r} \cap \Sigma}\left\langle x, \nabla^{\Sigma} h+h\left(\mathbf{H}_{\mathbf{f}}-\bar{\nabla} f\right)\right\rangle d_{f} v \geq-\frac{r}{\omega_{k}} \int_{B_{r} \cap \Sigma}\left|\nabla^{\Sigma} h\right|+h\left|\mathbf{H}_{\mathbf{f}}-\bar{\nabla} f\right| d_{f} v .
$$


Integrating on $0<\sigma<\rho$, we get

$$
J(\sigma) \leq J(\rho)+\int_{\sigma}^{\rho}\left(\frac{r^{-k}}{\omega_{k}} \int_{B_{r} \cap \Sigma}\left|\nabla^{\Sigma} h\right|+h\left|\mathbf{H}_{\mathbf{f}}-\bar{\nabla} f\right| d_{f} v\right) d r .
$$

Now, set

$$
\phi(\rho)=\frac{1}{\omega_{k}} \int_{B_{\rho} \cap \Sigma} h d_{f} v \text { and } \psi(\rho)=\frac{1}{\omega_{k}} \int_{B_{\rho} \cap \Sigma}\left[\left|\nabla^{\Sigma} h\right|+h\left|\mathbf{H}_{\mathbf{f}}-\bar{\nabla} f\right|\right] d_{f} v .
$$

If $y \in \Sigma$ is a point with $h(y) \geq 1$, then using (4.9) we have that our choice of $\phi$ and $\psi$ implies that (4.1) and (4.2) hold. Furthermore,

$$
\rho_{0}=2 \phi_{\infty}^{1 / k}
$$

where

$$
\phi_{\infty}=\lim _{\rho \rightarrow \infty} \phi(\rho)=\frac{1}{\omega_{k}} \int_{\Sigma} h d_{f} v<\infty,
$$

because $h$ has compact support. So, applying Lemma 4.2 we have that there exists $\rho_{y} \in\left(0, \rho_{0}\right)$ such that

$$
\phi\left(5 \rho_{y}\right) \leq \frac{5^{k}}{2} \rho_{0} \psi\left(\rho_{y}\right)
$$

i.e,

$$
\int_{B_{5 \rho_{y}} \cap \Sigma} h d_{f} v \leq \frac{5^{k}}{w_{k}^{1 / k}}\left(\int_{\Sigma} h d_{f} v\right)^{1 / k} \int_{B_{\rho} \cap \Sigma}\left[\left|\nabla^{\Sigma} h\right|+h\left|\mathbf{H}_{\mathbf{f}}-\bar{\nabla} f\right|\right] d_{f} v .
$$

Let $S \subset \Sigma$ be the set where $h \geq 1$, that is,

$$
S=\{y \in \Sigma \mid h(y) \geq 1\} .
$$

Using Covering Lemma 4.1, we can choose disjoint balls $B^{j}$ satisfying (4.10) so that

$$
S \subset \bigcup_{j} 5\left(B^{j}\right)
$$

It follows that

$$
\int_{S} h d_{f} v \leq \frac{5^{k}}{w_{k}^{1 / k}}\left(\int_{\Sigma} h d_{f} v\right)^{1 / k} \int_{\Sigma}\left[\left|\nabla^{\Sigma} h\right|+h\left|\mathbf{H}_{\mathbf{f}}-\bar{\nabla} f\right|\right] d_{f} v .
$$

Fix some $\epsilon>0$ and a monotone function $\gamma(t)$ with

$$
\gamma(t)= \begin{cases}1 & \text { if } t \geq \epsilon \\ 0 & \text { if } t \leq 0\end{cases}
$$

Given any $t>0$, we set

$$
A_{t}=\{x \in \Sigma \mid u(x)>t\} .
$$

Let $\mu$ be the volume measure on $\Sigma$. Using $h(x)=\gamma(u(x)-t)$ in (4.12), we have

$$
\begin{aligned}
\mu\left(A_{t+\epsilon}\right) & \leq \frac{5^{k}}{w_{k}^{1 / k}}\left[(t+\epsilon)^{\frac{k}{k-1}} \mu\left(A_{t}\right)\right]^{1 / k} \int_{\Sigma}\left[\left|\nabla^{\Sigma} u\right| \gamma^{\prime}(u-t)+\gamma(u-t)\left|\mathbf{H}_{\mathbf{f}}-\bar{\nabla} f\right|\right] \\
& \leq \frac{5^{k}}{w_{k}^{1 / k}}\left(\int_{\Sigma}(u+\epsilon)^{\frac{k}{k-1}}\right)^{1 / k} \int_{\Sigma}\left|\nabla^{\Sigma} u\right| \gamma^{\prime}(u-t)+\int_{A_{t}}\left|\mathbf{H}_{\mathbf{f}}-\bar{\nabla} f\right| .
\end{aligned}
$$


Multiplying by $(t+\epsilon)^{\frac{1}{k-1}}$ gives

$$
(t+\epsilon)^{\frac{1}{k-1}} \mu\left(A_{t+\epsilon}\right) \leq \frac{5^{k}}{w_{k}^{1 / k}}\left(\mu\left(A_{t}\right)\right)^{1 / k} \int_{\Sigma}\left[\left|\nabla^{\Sigma} u\right| \gamma^{\prime}(u-t)+\gamma(u-t)\left|\mathbf{H}_{\mathbf{f}}-\bar{\nabla} f\right|\right] .
$$

Integrating the inequality above in $t$ and using Lemma 4.3 with $\nu=\mu, t_{0}=\epsilon$, and $\alpha-1=\frac{1}{k-1}$, so that $\alpha=\frac{k}{k-1}$ in the first term gives

$$
\begin{aligned}
\frac{k-1}{k} \int_{A_{\epsilon}}\left(u^{\frac{k}{k-1}}-\epsilon^{\frac{k}{k-1}}\right) \leq & \frac{5^{k}}{w_{k}^{1 / k}}\left(\int_{\Sigma}(u+\epsilon)^{\frac{k}{k-1}}\right)^{1 / k} \\
& \cdot \int_{0}^{\infty}\left(\int_{\Sigma}\left|\nabla^{\Sigma} u\right| \gamma^{\prime}(u-t)+\int_{A_{t}}\left|\mathbf{H}_{\mathbf{f}}-\bar{\nabla} f\right|\right) \\
= & \frac{5^{k}}{w_{k}^{1 / k}}\left(\int_{\Sigma}(u+\epsilon)^{\frac{k}{k-1}}\right)^{1 / k} \int_{\Sigma}\left(\left|\nabla^{\Sigma} u\right|+u\left|\mathbf{H}_{\mathbf{f}}-\bar{\nabla} f\right|\right),
\end{aligned}
$$

where the equality used the fundamental theorem of calculus to evaluate the $\gamma^{\prime}$ term and Lemma 4.3 with $\nu=\left|\mathbf{H}_{\mathbf{f}}-\bar{\nabla} f\right| \mu, t_{0}=0$ and $\alpha=1$ to evaluate the last term. Letting $\epsilon \rightarrow 0$, we obtain the estimate with

$$
c_{k}=\frac{k}{k-1} \frac{5^{k}}{w_{k}^{1 / k}} .
$$

The other associated inequality comes from Theorem 1.2 and Hölder's inequality as we will see next.

Corollary 4.6. There exists $c_{k}$ depending only on $k$ so that if $u \geq 0$ is a Lipschitz function with compact support on a submanifold $\Sigma^{k} \subset \mathbb{R}_{f}^{n}$ and $p \in[1, k)$, then

$$
\|u\|_{L^{p^{*}}} \leq c_{k}\left(\frac{(k-1) p}{k-p}\left\|\nabla^{\Sigma} u\right\|_{L^{p}}+\left\|u\left(\mathbf{H}_{\mathbf{f}}-\bar{\nabla} f\right)\right\|_{L^{p}}\right),
$$

where $p^{*}=\frac{k p}{k-p}$.

The proof follows as in Corollary 3.12 in 3 .

As an immediate consequence of the Sobolev inequality we have the following general form of the isoperimetric inequality.

Proposition 4.7. Let $\Omega \subset \Sigma$ be open with Lipschitz boundary $\partial \Omega$. Then we have the inequality

$$
V_{f}(\Omega)^{\frac{k-1}{k}} \leq c_{k}\left[V_{f}(\partial \Omega)+\int_{\Omega}\left|\mathbf{H}_{\mathbf{f}}-\bar{\nabla} f\right|\right] .
$$

To see that the isoperimetric inequality follows the weighted Michael-Simon Inequality, we take a suitable sequence of nonnegative $C_{c}^{1}$-functions on $\Sigma$ which approximates the characteristic function of an open set $\Omega \subset \Sigma$ and apply the Sobolev Inequality for them.

\section{Diameter CONTrol}

To finish this work, we present one application of the weighted Michael-Simon inequality. We prove an estimate for the diameter depending on the $f$-mean curvature. This section is based on the work of Topping [16]. 
Lemma 5.1. Let $\Sigma^{k} \hookrightarrow \mathbb{R}_{f}^{n}$ be a submanifold. If (1.2) occurs, then there exists a constant $\delta(n, f)>0$ such that for any $x \in \Sigma$ and $R>0$, at least one of the following two alternatives occurs:

(1) $M_{f}(x, R)>\delta$;

(2) $\kappa_{f}(x, R)>\delta$,

where

$$
M_{f}(x, R):=\sup _{r \in(0, R]} r^{-\frac{1}{k-1}}\left[V_{f}(x, r)\right]^{-\frac{k-2}{k-1}} \int_{B_{r}(x)}\left|\mathbf{H}_{\mathbf{f}}\right| d_{f} v
$$

is the maximal function, and

$$
\kappa_{f}(x, R):=\inf _{(0, R]} \frac{V_{f}(x, r)}{r^{k}} .
$$

is the measure collapsedness rate.

Proof. Given a $\delta>0$ to be chosen later, suppose that $M_{f}(x, R) \leq \delta$. Then, for all $r \in(0, R]$, we have

$$
\int_{B_{r}(x)}\left|\mathbf{H}_{\mathbf{f}}\right| d_{f} v \leq \delta r^{\frac{1}{n-1}}\left[V_{f}(x, r)\right]^{\frac{n-2}{n-1}} .
$$

Given a fixed $x$, let $V_{f}(r):=V_{f}(x, r)$. So, as the volume without weight is differentiable, whe have that $V_{f}(v)$ is differenciable a.e.w. $r>0$. For such $r \in(0, R]$, and any $\lambda>0$, define a Lipschitz cut-off function $u: \Sigma \rightarrow \mathbb{R}$ by

$$
u(y)= \begin{cases}1, & y \in B_{r}(x), \\ 1-\frac{1}{\lambda}\left(\operatorname{dist}_{\Sigma}(x, y)-r\right), & y \in B_{r+\lambda}(x) \backslash B_{r}(x), \\ 0 & y \notin B_{r+\lambda}(x) .\end{cases}
$$

Now, applying the weighted Michel-Simon inequality, we get

$$
\begin{aligned}
V_{f}(r)^{\frac{k-1}{k}} & \leq\left(\int_{B_{r+\lambda}} u^{\frac{k}{k-1}} d_{f} v\right)^{\frac{k-1}{k}} \\
& \leq \frac{c_{k}}{\lambda}\left(V_{f}(r+\lambda)-V_{f}(r)\right)+c_{k}\left\|\mathbf{H}_{\mathbf{f}}\right\|_{L^{1}\left(B_{r+\lambda}(x)\right)} .
\end{aligned}
$$

Letting $\lambda \rightarrow 0$, we have

$$
V_{f}(r)^{\frac{k-1}{k}} \leq c_{k} \frac{d V_{f}}{d r}+c_{k}\left\|\mathbf{H}_{\mathbf{f}}\right\|_{L^{1}\left(B_{r}(x)\right)} .
$$

Using (5.1), we obtain

$$
c_{k} \frac{d V_{f}}{d r}+c_{k} \delta r^{\frac{1}{k-1}}\left[V_{f}(x, r)\right]^{\frac{k-2}{k-1}}-V_{f}(r)^{\frac{k-1}{k}} \geq 0 .
$$

Now, we choose $\delta>0$ that to be sufficiently small so that $\delta<C w_{k}$, where $C=$ $\inf _{B_{r}}\left\{e^{-f}\right\}$ and $w_{k}$ is the volume of the unit ball in $\mathbb{R}^{k}$, and define the function $v(r):=\delta r^{k}$. Computing as in Topping [16], for $\delta$ sufficiently small, we have

$$
c_{k} \frac{d v_{f}}{d r}+c_{k} \delta r^{\frac{1}{k-1}}[v(r)]^{\frac{k-2}{k-1}}-v(r)^{\frac{k-1}{k}} \leq 0 .
$$

Then, using (5.1), (5.3) and the fact that

$$
\frac{V_{f}(r)}{r^{k}} \geq \frac{C w_{k} V_{f}(r)}{V_{f}\left(B_{r}\right)} \rightarrow C w_{k}
$$


as $r \rightarrow 0$, while $v(r) / r^{k}=\delta<C w_{k}$, we deduce that $V_{f}(r)>v(r)$ for all $r \in(0, R]$ and hence

$$
\kappa_{f}(x, R)=\inf _{r \in(0, R]} \frac{V_{f}(x, r)}{r^{k}}>\delta,
$$

as desired.

In possession of the previous lemma we are able to prove Theorem 1.4

Proof of Theorem 1.4. Choose $R>0$ such that $V(\Sigma)<\delta R^{k}$, where $V(\Sigma)$ is the total volume of $\Sigma$ and $\delta$ is given by Lemma 5.1. In particular, for all $z \in \Sigma$, we have

$$
\kappa_{f}(z, R) \leq \frac{V_{f}(z, R)}{R^{k}} \leq \delta .
$$

So, by Lemma 5.1, we must have that $M_{f}(z, R)>\delta$. Therefore, by definition of the maximal function and the Holder inequality, we have that there exists $r=r(z)$ such that

$$
\begin{aligned}
\delta & <r^{-\frac{1}{k-1}} V_{f}(z, r)^{-\frac{n-2}{n-1}} \int_{B_{r}(z)}\left|\mathbf{H}_{\mathbf{f}}\right| d_{f} v \\
& \leq r^{-\frac{1}{k-1}}\left(\int_{B_{r}(z)}\left|\mathbf{H}_{\mathbf{f}}\right|^{k-1} d_{f} v\right)^{\frac{1}{k-1}}
\end{aligned}
$$

and hence

$$
r(z) \leq \delta^{1-k} \int_{B_{r(z)}(z)}\left|\mathbf{H}_{\mathbf{f}}\right|^{k-1} d_{f} v .
$$

Now let $x, y \in \Sigma$ be any two extremal points, by which we mean that $d_{\text {int }}=$ $\operatorname{dist}_{\Sigma}(x, y)$, and let $\gamma \subset \Sigma$ be any shortest geodesic connecting $x$ and $y$. The set of balls $B_{r(z)}(z)$ with $z \in \gamma$ is clearly a covering of $\gamma$. Then, using the same covering argument as in Topping [16], for any $\lambda \in\left(0, \frac{1}{2}\right)$, we can find a sequence of points $\left\{z_{i}\right\} \subset \gamma$ such that the balls $\left\{B_{r\left(z_{i}\right)}\left(z_{i}\right)\right\}$ are disjoint and cover at least a fraction $\lambda$ of the whole of $\Sigma$ :

$$
\lambda d_{\text {int }} \leq \sum_{i} 2 r\left(z_{i}\right)
$$

Joining this with (5.5), we obtain

$$
\begin{aligned}
d_{i n t} & \leq \frac{2}{\lambda} \sum_{i} r\left(z_{i}\right) \leq \frac{2}{\lambda} \delta^{1-k} \sum_{i} \int_{B_{r\left(z_{i}\right)}\left(z_{i}\right)}\left|\mathbf{H}_{\mathbf{f}}\right|^{k-1} d_{f} v \\
& \leq \frac{2}{\lambda} \delta^{1-k} \int_{\Sigma}\left|\mathbf{H}_{\mathbf{f}}\right|^{k-1} d_{f} v .
\end{aligned}
$$

This finishes the proof of Theorem 1.4

\section{REFERENCES}

[1] William K. Allard, On the first variation of a varifold, Ann. of Math. (2) 95 (1972), 417-491. MR0307015 (46 \#6136)

[2] E. Bombieri, E. De Giorgi, and M. Miranda, Una maggiorazione a priori relativa alle ipersuperfici minimali non parametriche (Italian), Arch. Rational Mech. Anal. 32 (1969), 255-267. MR.0248647 (40 \#1898)

[3] Tobias Holck Colding and William P. Minicozzi II, A course in minimal surfaces, Graduate Studies in Mathematics, vol. 121, American Mathematical Society, Providence, RI, 2011. MR 2780140 
[4] Tobias H. Colding and William P. Minicozzi II, Generic mean curvature flow I: generic singularities, Ann. of Math. (2) 175 (2012), no. 2, 755-833, DOI 10.4007/annals.2012.175.2.7. MR.2993752

[5] David Hoffman and Joel Spruck, Sobolev and isoperimetric inequalities for Riemannian submanifolds, Comm. Pure Appl. Math. 27 (1974), 715-727. MR0365424 (51 \#1676)

[6] D. Impera and M. Rimoldi, Stability properties and topology at infinity of f-minimal hypersurfaces. arXiv:1302.6160 [math.DG].

[7] Jia-Yong Wu and Yu Zheng, Relating diameter and mean curvature for Riemannian submanifolds, Proc. Amer. Math. Soc. 139 (2011), no. 11, 4097-4104, DOI 10.1090/S0002-99392011-10848-7. MR2823054(2012e:53120)

[8] V. Maz'ya, (Editor) Sobolev spaces in Mathematics I, II, II.

[9] J. H. Michael and L. M. Simon, Sobolev and mean-value inequalities on generalized submanifolds of $R^{n}$, Comm. Pure Appl. Math. 26 (1973), 361-379. MR0344978 (49 \#9717)

[10] Mario Miranda, Diseguaglianze di Sobolev sulle ipersuperfici minimali (Italian), Rend. Sem. Mat. Univ. Padova 38 (1967), 69-79. MR0221350 (36 \#4402)

[11] M. Batista and H. Mirandola, A Sobolev-type inequality for submanifolds in weighted Riemannian Manifolds, arXiv:1304.2271 [math.DG].

[12] Nam Q. Le, Blow up of subcritical quantities at the first singular time of the mean curvature flow, Geom. Dedicata 151 (2011), 361-371, DOI 10.1007/s10711-010-9538-z. MR2780756 (2012m:53142)

[13] S. L. Soboleve, On a theorem of functional analysis (Russian). Mat. Sb. 4, no.3 (1938), 471-496. English trans.: Eleven papers in Analysis. Am. Math. Transl. (2) 34, (1963), 39-68.

[14] Gu-Ji Tian and Xu-Jia Wang, A class of Sobolev type inequalities, Methods Appl. Anal. 15 (2008), no. 2, 263-276, DOI 10.4310/MAA.2008.v15.n2.a10. MR2481683 (2010e:35016)

[15] Peter Topping, Diameter control under Ricci flow, Comm. Anal. Geom. 13 (2005), no. 5, 1039-1055. MR2216151 (2006m:53101)

[16] Peter Topping, Relating diameter and mean curvature for submanifolds of Euclidean space, Comment. Math. Helv. 83 (2008), no. 3, 539-546, DOI 10.4171/CMH/135. MR2410779 (2009b:53100)

[17] Qiaoling Wang, Complete submanifolds in manifolds of partially non-negative curvature, Ann. Global Anal. Geom. 37 (2010), no. 2, 113-124, DOI 10.1007/s10455-009-9176-6. MR:2578260(2011a:53115)

[18] William P. Ziemer, Weakly differentiable functions, Graduate Texts in Mathematics, vol. 120, Springer-Verlag, New York, 1989. Sobolev spaces and functions of bounded variation. MR1014685(91e:46046)

Departamento de Matemática, Universidade Federal da Paraíba, 58051-900, João PesSOA, PB, BRAZIL

E-mail address: adrianoalves@mat.ufpb.br 